\title{
Automated Fast-Flow Synthesis of Peptide Nucleic Acid Conjugates
}

\author{
Chengxi Li, Alex J. Callahan, Kruttika-Suhas Phadke, Bryan Bellaire, Charlotte E. Farquhar, Genwei Zhang, Carly K. \\ Schissel, Alexander J. Mijalis, Nina Hartrampf, Andrei Loas, David E. Verhoeven, and Bradley L. Pentelute*
}

\begin{abstract}
Peptide nucleic acids (PNAs) are charge-neutral oligonucleotides with emerging potential for treatment of genetic, acquired, and viral diseases, including COVID-19. Their challenging synthesis, however, limits their use for rapid therapeutic intervention and widespread application. Here, we report a highly efficient technology that utilizes a fully automated fast-flow instrument to manufacture cell-penetrating peptide-conjugated PNAs (PPNAs) in a single shot. The machine is rapid: each amide bond is formed in 10 seconds and the synthesis of an 18-mer bioactive PNA is complete in one hour. Anti-IVS2-654 PPNA synthesized in a single shot with this instrument presented over 16-fold activity compared to unmodified PNA in a splice-correction assay. We demonstrated the utility of this approach by chemically synthesizing an eight-member anti-SARS-CoV-2 PPNA library within one day. A designer PPNA targeting the 5' untranslated region of SARS-CoV-2 genomic RNA reduced the viral titer by over $95 \%$ in live virus infection assays $\left(\mathrm{IC}_{50}: 0.8 \mu \mathrm{M}\right)$. Our technology can rapidly yield on-demand PPNA candidates to tackle newly-emerging viral pathogens.
\end{abstract}

\section{INTRODUCTION}

Peptide nucleic acids (PNAs) are artificial singlestranded DNA-like molecules in which the sugar-phosphodiester moiety is replaced with an uncharged $N$-(2-aminoethyl) glycine unit and the nucleobases are attached via a methyl carbonyl linker. ${ }^{1}$ Due to the attenuated electrostatic repulsion in PNA-DNA/RNA duplexes, PNAs can hybridize complementary DNA and RNA with higher affinity and specificity than DNA-DNA/RNA duplexes. ${ }^{2}$ Additionally, the amidebased PNA backbone offers unique physiochemical properties including superior chemical, thermal, and enzymatic stability. ${ }^{3}$

PNAs have found a wide range of chemical and biological applications, ${ }^{4}$ especially as antisense agents in developing gene-specific therapeutics for various diseases including cancer ${ }^{5}$ and monogenic blood disorders ${ }^{6}$ as novel antibiotics, ${ }^{4 c}$, ${ }^{7}$ and as antivirals. ${ }^{8}$ As the causative agent of coronavirus disease 2019 (COVID-19), the severe acute respiratory syndrome coronavirus 2 (SARS-CoV-2) ${ }^{9}$ is a pathogen of immense importance to global public health. Development of innovative direct-acting antiviral agents is sorely needed to address this virus. Antisense PNAs are expected to inhibit the SARS-CoV-2 replication by reducing the expression of targeted RNA through sequence-specific steric blocking (Fig. 1a), which have been proved effective against SARS-CoV. . $^{8 \mathrm{a}}$

In order to find the most effective PNA sequence for a given indication, screening small libraries of PNA candidates in a high-throughput manner is crucial. The chemical synthesis of long target-specific PNA strands (Fig. 1b), remains a challenge, however. PNA synthesis is limited by strong on-resin aggregation and side-reactions including deletion, rearrangement, isomerization, and nucleobase additions result in low isolated yields of the desired product..$^{10}$ Most of the reported synthetic PNA sequences are limited to fewer than 15 residues, especially for purine-rich sequences. ${ }^{10}$ Efforts to improve synthetic efficiency are commonplace, such as extensive capping and frequent double-couplings, but with a great sacrifice of convenience. Therefore, automation technology ${ }^{11}$ is needed to improve the PNA synthesis efficiency and in the meanwhile, reduce its complexity. In previous studies, commercial DNA synthesizers could be updated for PNA synthesis, of which each coupling cycle takes 32 minutes, but additional capping and double-coupling are still needed to improve the synthesis efficiency. ${ }^{12}$ The current low-efficiency synthesis protocols hinder the rapid production of screening libraries needed for antisense PNA sequence optimization, and development timelines remain long.

To address this challenge, we report a highly efficient technology in which chemistry is matched with an automated fast-flow instrument that increases the PNA synthesis rate by over an order of magnitude with significantly improved crude sample purity (Fig. 1d). The new synthesis protocol only requires three minutes for the addition of each residue, compared with the reported $\sim 1 \mathrm{~h}$ manual protocols. ${ }^{13}$ An important variable temperature design increases coupling efficiency, while at the same time reducing on-resin aggregation and other side-reactions.

Due to the neutral charge of the PNA structure, intracellular delivery of PNAs is inefficient, but conjugating with cell-penetrating peptides (CPPs) can address this issue. Instead of using common stepwise synthesis ${ }^{14}$ via click chemistry ${ }^{15}$ (Fig. 1c), the high coupling efficiency reported here allows direct manufacture of PNA conjugates with cell-penetrating peptides (PPNAs) in a single-shot (Fig. 1d).

To demonstrate the reliability and applicability of our method, we synthesized a 33-residue PPNA which hybridizes to the $\beta$-thalassemia gene sequence, IVS2-654, ${ }^{16}$ for splice-correction activity. The enhanced green fluorescence protein (EGFP) assay ${ }^{16}$ showed that the single-shot synthesized PPNA presented over 16-fold activity compared to unmodified PNA. The power of this automated technology was further strengthened by the synthesis of an eight-member PPNA library (33residue each) targeting SARS-CoV-2 genomic RNA in a single day. One of the designed PPNAs targeting the 5' untranslated region (5'UTR) was highly efficacious, reducing viral titers by over $95 \%$ in live infection assays. Overall, we believe this platform could find broad application not only in generating new SARS-CoV-2 antisense therapeutics, but also for rapid development of PPNA candidates to treat other genetic diseases and emerging viral pathogens. 


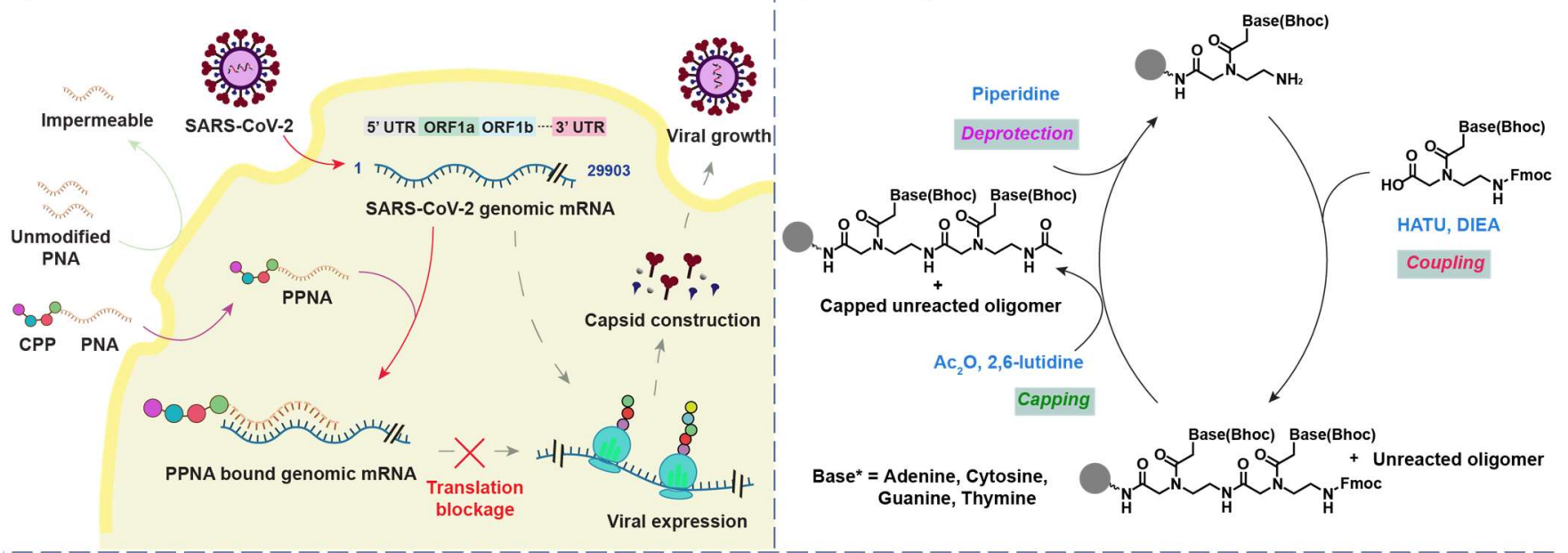

c) Manual Stepwise Synthesis of PPNA
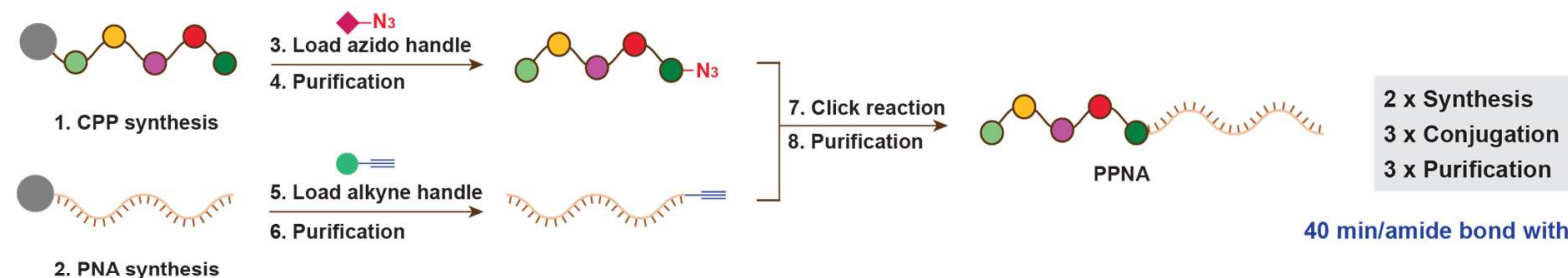

2. PNA synthesis

d) Automated Single-Shot Fast-flow Synthesis of PPNA (This work)

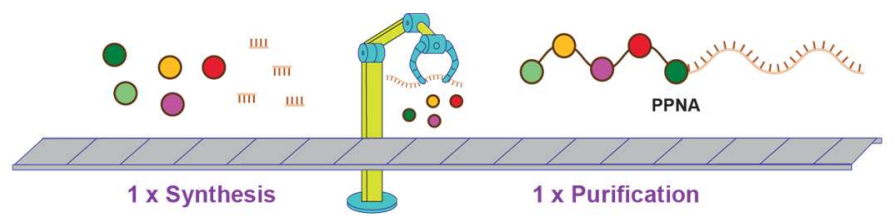

- Computer-controlled fully automated synthesis

- Single-shot access PPNAs

- 10 sec/amide bond without capping

- Significantly improved purity over manual protocols

- Over $95 \%$ inhibition of live SARS-CoV-2 infection using designed PPNAs

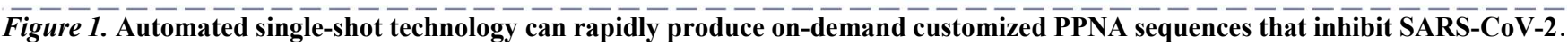
(a) An antisense PPNA binding to the 5' UTR of the SARS-CoV-2 genomic transcript can prevent the expression of viral genes and subsequently inhibit viral growth. (b) Typical coupling, deprotection, and capping procedures during PNA assembly in the solid phase. (c) Workflow for the manual stepwise synthesis of PPNAs with click chemistry. (d) An automated fast-flow synthesizer allows for rapid manufacture of PPNAs in a single-shot. Fmoc: 9-fluorenylmethoxycarbonyl; HATU: $O$-(7-azabenzotriazol-1-yl)-1,1,3,3-tetramethyluronium hexafluorophosphate. DIEA: N,N-Diisopropylethylamine. Ac $2 \mathrm{O}$ : acetic anhydride. Fmoc: 9-fluorenylmethoxycarbonyl. Bhoc: benzhydryloxycarbonyl.

\section{RESULTS AND DISCUSSION Automated Microscale Flow Synthesizer Design}

The automated flow PNA synthesizer consists of seven modules including a central control computer, solution storage system, three HPLC pumps, three multi-position valves, heating elements, reaction zone, and a UV-visible detector. A modular script in the Mechwolf programming environment ${ }^{17}$ controls the instrument (Fig. 2a). During a coupling reaction, three HPLC pumps draw reagents from the storage module under a nitrogen atmosphere and the desired PNA monomer, activator, and base solutions are mixed using a valve. The mixture flows through a module electrically heated at $70{ }^{\circ} \mathrm{C}$, forming an activated ester. The activated PNA monomer flows next through the reaction zone, a packed bed of resin maintained at $70{ }^{\circ} \mathrm{C}$, where amide bond formation is completed within $10 \mathrm{~s}$. During the deprotection step, the piperidine solution flows through a room temperature loop controlled by a multi-position valve (Fig. 2a) and meets with the $70{ }^{\circ} \mathrm{C}$ reactor to generate an $\sim 40{ }^{\circ} \mathrm{C}$ environment, which enables rapid and efficient deprotection with minimized nucleobase adducts. An in-line UVvisible detector was used to monitor the composition of the spent reagent solution (Fig. 2b). The coupling yield, deprotection efficiency, and the mass transfer rate through the resin can be inferred in real-time through the Fmoc-removal absorbance chromatogram. ${ }^{18}$ This modular and in-line detection design allows for fast chemistry optimization for not only PNAs but also other biopolymer syntheses.

The reusable reactor body (Fig. 2a) is designed for a $7.5 \mu \mathrm{mol}$ scale synthesis and is typically loaded with $15 \mathrm{mg}$ of $0.5 \mathrm{mmol} / \mathrm{g}$ Rink Amide resin. While reducing expensive monomer consumption, this design can deliver milligrams of pure product, which is often sufficient for subsequent biological characterizations. A detailed workflow and timeline for a typical synthesis process is listed in Fig. 2c, which presents the amide bond formation step and the overall solid-phase PNA synthesis cycle within three minutes. More instrument and synthesis details can be found in the supporting information section 3 to 7 . 
a)
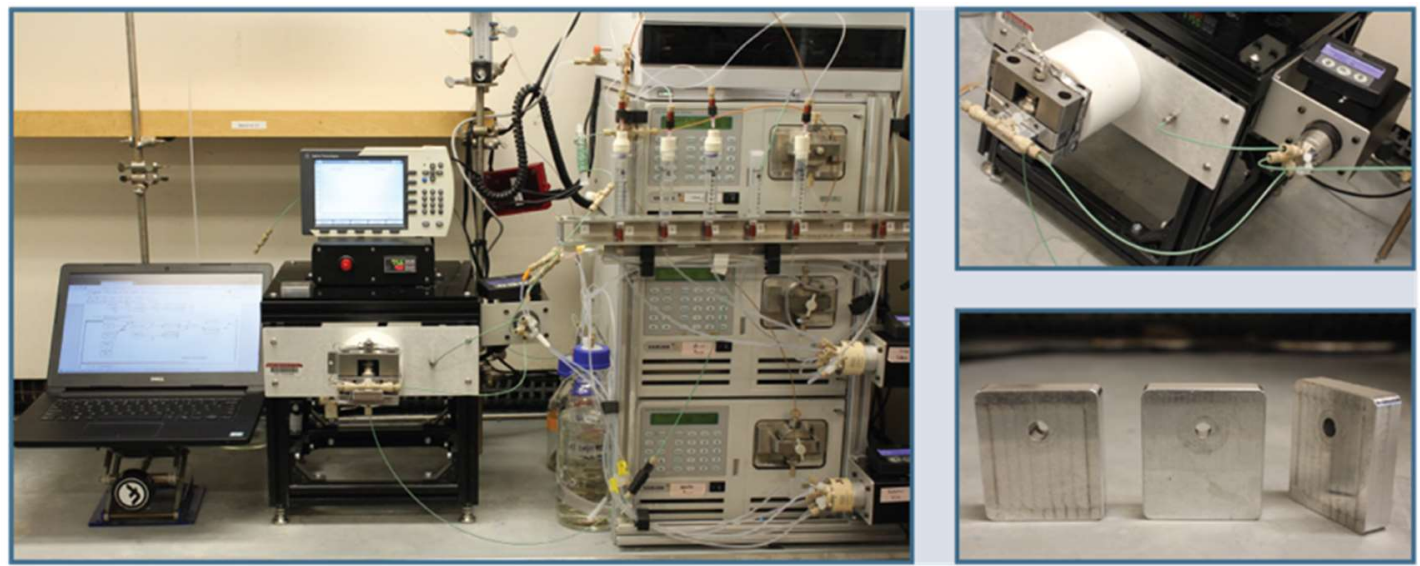

b)

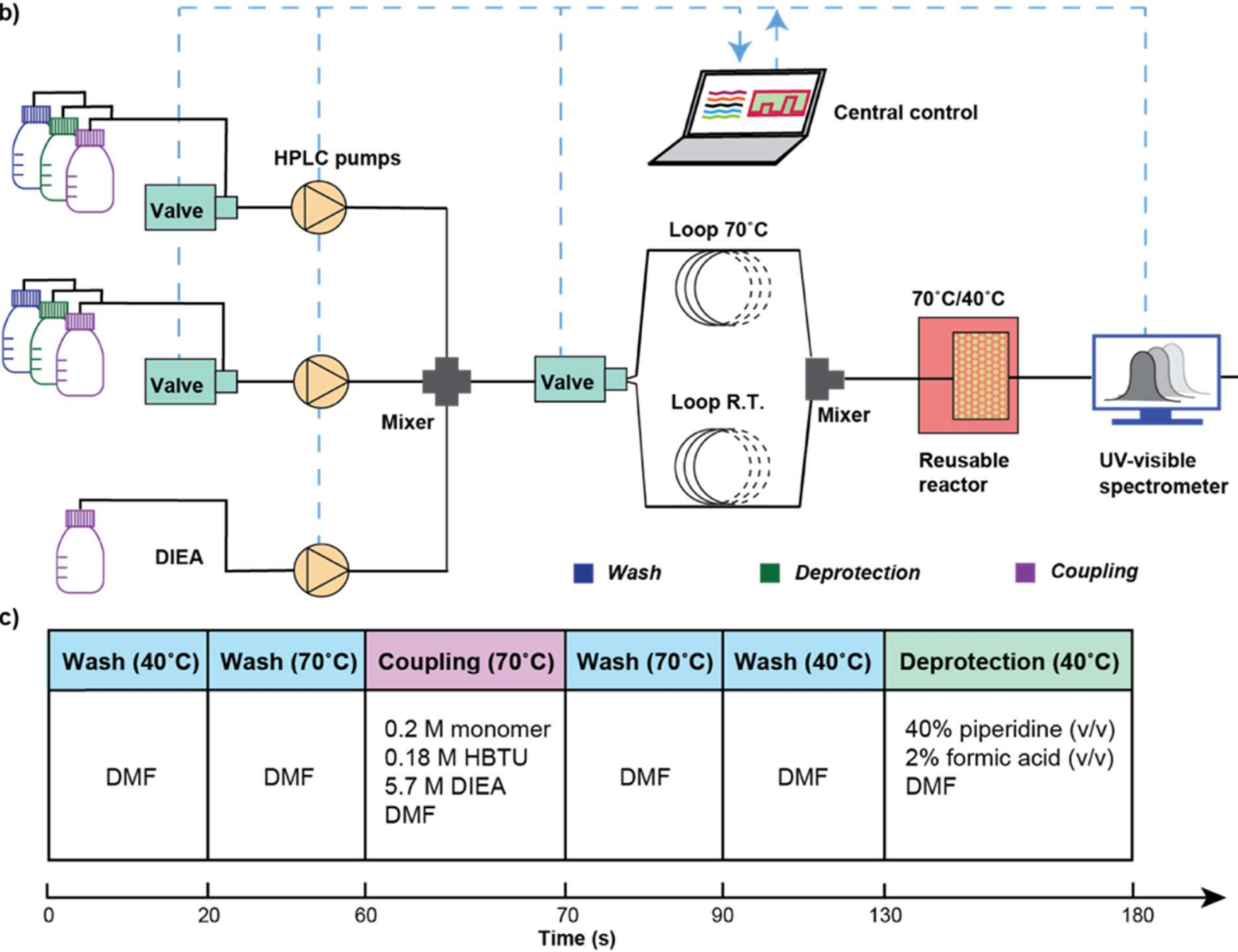

Figure 2. Automated flow PNA synthesis enables 10-second amide bond formation and complete solid-phase synthesis cycles in three minutes. (a) Photographs of the automated flow solid-phase synthesizer: full image, variant temperature valve, and reusable reactors. (b) Process flow diagram. Amino acids, activating agents, and DIEA are mixed together by three HPLC pumps. Two multi-position valves control the selection of the amino acids and activating agents. The third valve controls variant temperature flow paths. Amino acids are activated in the $70{ }^{\circ} \mathrm{C}$ loop, then flowed over the resin bed housed in a reusable reactor. The effluent is passed through a UV-visible spectrometer to waste. (c) Cycle diagram showing the duration of each step, the solution composition during each step after mixing, and the time used at each step. HBTU: $O$-(benzotriazol-1-yl)- $N, N, N^{\prime}, N^{\prime}$-tetramethyluronium hexafluorophosphate; DMF: $N, N$-dimethylformamide.

\section{Optimization of Automated PNA Synthesis}

In-line UV-visible monitoring combined with liquid chromatography-mass spectrometry (LC-MS) and high-performance liquid chromatography (HPLC) product characterization allows for rapid optimization of PNA synthesis conditions. We began the optimization with a manually synthesized 4-mer PNA in $\sim 4$ h of in-lab effort ${ }^{10,13}$ with $57 \%$ crude purity (Table 1 , entry 1). During the manual synthesis, base can promote transamidation of the nucleobase to the $\mathrm{N}$-terminus, resulting in an isomeric product $(15 \%)$, similarly, transamidation on the main chain can lead to formation of a ketopiperazine that gets washed away, causing deletion of the N-terminal PNA unit (4\%), and the benzhydryloxycarbonyl (Bhoc) protecting group can be cleaved by piperidine to yield a nucleobase adduct $(2 \%)$ (supporting information S6). Next, the same PNA sequence was synthesized on the automated synthesizer within $15 \mathrm{~min}$ at 70 ${ }^{\circ} \mathrm{C}$ (Table 1, entry 2 ). High temperature accelerated both on- 
Table 1. Evaluation of reaction conditions for the automated flow PNA synthesis ${ }^{a}$

\begin{tabular}{|c|c|c|c|c|c|c|c|c|c|c|}
\hline Entry & Activator & $\begin{array}{c}\text { Couple } \\
\text { temp. }\left({ }^{\circ} \mathrm{C}\right)\end{array}$ & Depro. sol. & $\begin{array}{l}\text { Depro. } \\
\left.\text { temp. ( }{ }^{\circ} \mathrm{C}\right)\end{array}$ & $\begin{array}{l}\text { Couple } \\
\text { time }\end{array}$ & $\begin{array}{l}\text { Purity } \\
\text { (\%) }\end{array}$ & $\begin{array}{l}\text { Isomer } \\
(\%)\end{array}$ & $\begin{array}{c}\text { Deletion }^{c} \\
\text { (\%) }\end{array}$ & $\begin{array}{c}\text { Base } \\
\text { add. (\%) }\end{array}$ & $\begin{array}{c}\text { Unknown } \\
(\%)\end{array}$ \\
\hline 1 & PyAOP & RT & $20 \%$ piperidine & RT & $30 \min$ & 57 & 15 & 4 & 2 & 22 \\
\hline 2 & PyAOP & 70 & $20 \%$ piperidine & 70 & $10 \mathrm{sec}$ & 70 & 9 & 10 & 7 & 4 \\
\hline 3 & PУAOP & 70 & $20 \%$ piperazine & 70 & $10 \mathrm{sec}$ & 70 & 7 & 9 & 4 & 10 \\
\hline 4 & PУAOP & 70 & $20 \%$ morpholine & 70 & $10 \mathrm{sec}$ & 61 & 12 & 8 & 8 & 11 \\
\hline 5 & PYAOP & 70 & $\begin{array}{l}20 \% \text { piperidine, } \\
1 \% \text { Formic acid }\end{array}$ & 70 & $10 \mathrm{sec}$ & 68 & 11 & 10 & 3 & 8 \\
\hline 6 & PYAOP & 70 & $\begin{array}{l}20 \% \text { piperidine, } \\
2 \% \text { Formic acid }\end{array}$ & 70 & $10 \mathrm{sec}$ & 65 & 11 & 11 & 2 & 11 \\
\hline 7 & PУAOP & 70 & $\begin{array}{l}20 \% \text { piperidine, } \\
1 \% \text { Formic acid }\end{array}$ & 40 & $10 \mathrm{sec}$ & 76 & 5 & 9 & $<1$ & 9 \\
\hline 8 & PУAOP & 80 & $\begin{array}{l}20 \% \text { piperidine, } \\
1 \% \text { Formic acid }\end{array}$ & 45 & $10 \mathrm{sec}$ & 65 & 8 & 9 & 2 & 16 \\
\hline 9 & PYAOP & 90 & $\begin{array}{l}20 \% \text { piperidine, } \\
1 \% \text { Formic acid }\end{array}$ & 50 & $10 \mathrm{sec}$ & 47 & 12 & 10 & 3 & 28 \\
\hline 10 & HATU & 70 & $\begin{array}{l}20 \% \text { piperidine, } \\
1 \% \text { Formic acid }\end{array}$ & 40 & $10 \mathrm{sec}$ & 81 & 5 & 9 & $<1$ & 4 \\
\hline 11 & HBTU & 70 & $\begin{array}{l}20 \% \text { piperidine, } \\
1 \% \text { Formic acid }\end{array}$ & 40 & $10 \mathrm{sec}$ & 90 & $<1$ & 6 & $<1$ & 3 \\
\hline
\end{tabular}

${ }^{a}$ The PNA sequence: ACTG-Gly-CONH 2 . Conditions: manual synthesis: $100 \mathrm{mg}$ Rink Amide resin $(0.18$ mmol/g), 6 eq PNA monomer, 5.8 eq PyAOP, 6 eq DIEA, and 6 eq 2,5-lutidine in DMF. Automated flow synthesis: $15 \mathrm{mg}$ Rink Amide resin $(0.5 \mathrm{mmol} / \mathrm{g}), 10 \mathrm{eq}$ PNA monomer, 9.6 eq activator, 30 eq DIEA in DMF, flow rate: $2.5 \mathrm{~mL} / \mathrm{min}$. See supporting information for details. ${ }^{b}$ The crude purity was tracked with HPLC UV absorbance at $280 \mathrm{~nm}$. ${ }^{c}$ Total deletions of A, C, T, G, and Gly were summed. PyAOP: (7-Azabenzotriazol-1-yloxy)trispyrrolidinophosphonium hexafluorophosphate.

target $(70 \%)$ and off-target reactions, especially nucleobase adducts $(7 \%)$ and deletions $(10 \%)$. Further base screening, including piperazine and morpholine, revealed that piperidine was optimal for the Fmoc-deprotection at $70{ }^{\circ} \mathrm{C}$ (Table 1, entry 3-4).

A major focus of the high temperature optimization was prevention of piperidine adducts during the deprotection step. Addition of formic acid to deprotection solutions to prevent base-mediated aspartimide formation is a common practice, ${ }^{19}$ but we found the lower basicity is also useful in preventing nucleobase adduct formation. Formic acid ( $1 \%$ solution in $20 \%$ piperidine-DMF, v/v) was therefore used as an additive for deprotection and successfully decreased the base-adduct ratio to $3 \%$ (Table 1 , entry 5 ). Higher formic acid concentration can further inhibit the formation of base adducts but results in additional impurities (Table 1, entry 6). The most effective strategy, however, was to decrease the deprotection temperature to $40{ }^{\circ} \mathrm{C}$, which was controlled by a multi-position valve and a " $\mathrm{T}$ " connector (Fig. 2a). Under this condition, only negligible nucleobase adducts $(<1 \%)$ were generated (Table 1, entry 7 ).

Higher coupling temperature $\left(80{ }^{\circ} \mathrm{C}\right.$ and $\left.90{ }^{\circ} \mathrm{C}\right)$ can cause increased impurities ( $16 \%$ and $28 \%$, respectively), which indicates the highest allowable synthesis temperature is $70{ }^{\circ} \mathrm{C}$ on this flow instrument (Table 1, entry 8-9). A significant increase in purity $(81 \%)$ was achieved by using HATU as an activator, but $5 \%$ isomerization was still observed (Table 1, entry 10). Finally, the best condition was uncovered while using HBTU instead of HATU, which provided the cleanest PNA $(90 \%$ purity) with $<1 \%$ isomers and $<1 \%$ nucleobase adducts (Table 1 , entry 11$)$.

\section{Automated Single-Shot Synthesis of Potential Therapeutic PPNA}

The optimized high-temperature flow recipe provides PNA sequences with significantly improved purity to manual protocols in a fraction of the time. As depicted in Fig. 3, using batch protocols, production of a 4-mer PNA was completed in $4 \mathrm{~h}$. In comparison, our flow protocol provided the same sequence in only $15 \mathrm{~min}$ with superior purity $(90 \%$ vs $57 \%$, respectively) as demonstrated by both HPLC and LC-MS chromatograms (Fig. 3a to 3d).

To further test the synthetic efficiency of this methodology, an 18-mer PNA with a three-lysine $\left(\mathrm{K}_{3}\right)$ linker, ${ }^{20}$ which hybridizes to the $\beta$-thalassemia gene sequence (IVS2-654), was prepared in about one hour with $60 \%$ purity. We purified $4 \mathrm{mg}$ of pure PNA with $>95 \%$ purity for further biological characterizations (Fig. 3e, 3f).

This efficient protocol allows for single-shot synthesis of PPNAs. Conjugating cell-penetrating peptides (CPPs) with PNAs through click chemistry ${ }^{15}$ is a general strategy for PNA intracellular delivery. ${ }^{21}$ However, this method can be labor-intensive (Fig. 1c), and requires no human errors during the whole synthesis process. Also, multiple purifications can result in high sample losses. To expedite PPNA preparation, we developed a single-shot strategy to produce PPNAs using our automated platform. A 12-mer CPP "RXRRBRRXRRBR-CONH ${ }_{2}$ " (Bpep, $\mathrm{X}=6$-aminohexanoic acid; $\mathrm{B}=\beta$-alanine $)^{22}$ was pre-synthesized onto the resin on a peptide synthesizer developed previously in our lab, ${ }^{23}$ and the Bpep-loaded resin was transferred directly for PPNA synthesis on our microscale instrument. In 
this way, an anti-IVS2-654 PPNA was synthesized within $1.7 \mathrm{~h}$ with $52 \%$ crude purity. After purification, $1.2 \mathrm{mg}$ of pure material ( $>95 \%$ purity) were obtained (Fig. $3 \mathrm{~g}, 3 \mathrm{~h}$ ). This automated protocol not only achieved rapid synthesis but also enabled production of challenging PNA sequences that are difficult or unfeasible to synthesize in batch.

Manual Synthesis ACTG-Gly-CONH2
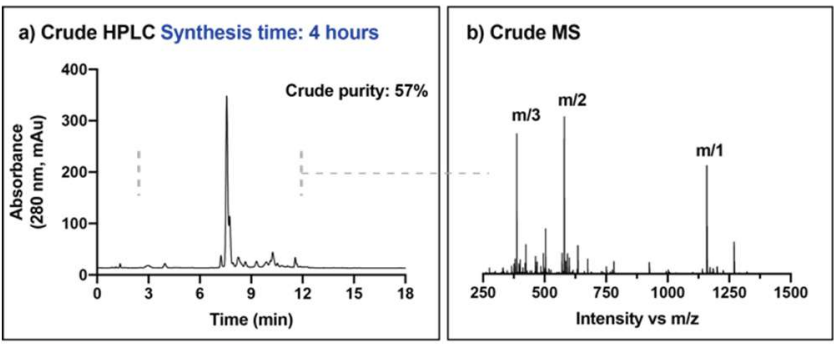

Automated Flow Synthesis ACTG-Gly-CONH2
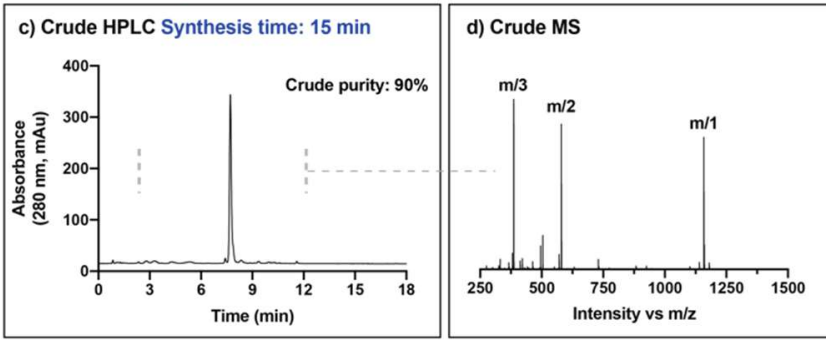

Automated Flow Synthesis GCTATTACCTTAACCCAG-KKK-CONH2

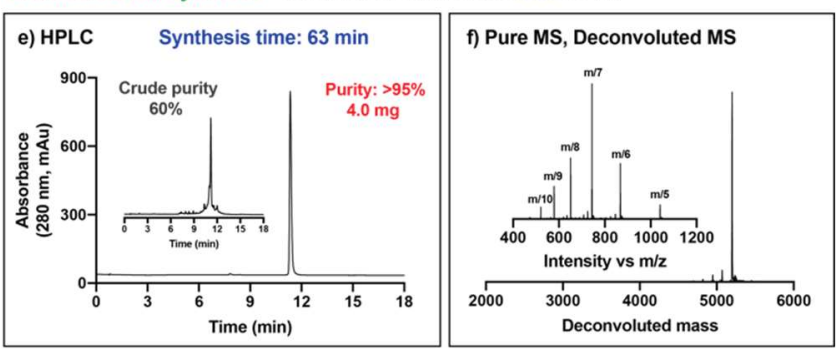

Automated Single-Shot Synthesis GCTATTACCTTAACCCAG-KKK-RXRRBRRXRRBR-CONH2
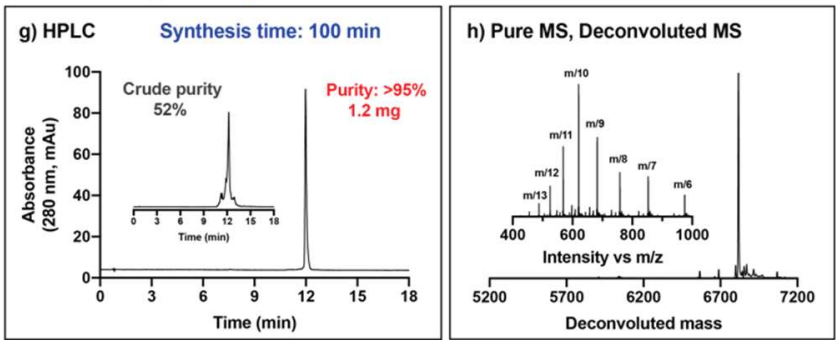

Figure 3. Automated fast-flow synthesis of PNAs and PPNAs with enhanced purity over manual protocols. (a-d) Crude HPLC/LC-MS traces of manually and flow synthesized 4-mer PNA. (e-f) Crude/pure HPLC, pure LC-MS traces of flow synthesized anti-IVS2-654 PNA. (g-h) Crude/pure HPLC, pure LC-MS traces of a single-shot synthesized anti-IVS2-654 PPNA.

\section{Synthetic anti-IVS2-654 PPNA Shows Enhanced Activity in an EGFP Assay}

The bioactivity of synthesized PPNAs was demonstrated by an enhanced green fluorescent protein (EGFP) assay in HeLa 654 cells. ${ }^{16}$ In this assay, the HeLa cells are stably engineered with an EGFP-coding sequence interrupted by an intron from the human $\beta$-globin gene (IVS2-654). The intron contains a cryptic splice site that leads to retention of a $\beta$-globin fragment in the EGFP mRNA sequence, resulting in the translation of a non-fluorescent protein. The anti-IVS2-654 PNA hybridizes to the aberrant $\beta$-globin 5 ' splice site, forcing the splicing machinery to use the normal splice sites and producing a functional, fluorescent EGFP. The PNA activity in the nucleus is therefore correlated with EGFP fluorescence and can be analyzed using flow cytometry, reported here as mean fluorescence intensity (MFI) relative to Phosphate-Buffered Saline (PBS) vehicle-treated cells. We used the synthesis platform for rapid production of an 18-mer anti-IVS2-654 PNA, a scramble 18-mer PNA (negative control), and an anti-IVS2-654 PPNA with milligram scales in $>95 \%$ purity.

A commercial anti-IVS2-654 PNA was obtained from PNA Bio ${ }^{\circledR}$ as a positive control (Fig. 4a). As depicted in Fig. $4 \mathrm{~b}$, the synthesized PNA and commercial PNA, with delivery mediated by lipofectamine, present similar activity in the EGFP assay, confirming function of the flow-produced material. The fluorescence did not increase when cells were treated with the scramble PNA, indicating sequence-dependent activity (Fig. 4b). Importantly, the single-shot synthesized PPNA improved the activity by over 16 -fold at $10 \mu \mathrm{M}$ compared to unmodified PNA (Fig. 4c). This result not only highlights the importance of cell-penetrating peptides but also demonstrates the potential to quickly build an active PPNA library for further applications.

\section{Synthetic anti-SARS-CoV-2 PPNA Shows Over 95\% Viral Inhibition in a Live Infection Assay}

The 5' UTR of the coronavirus genome is responsible for important biological functions, such as viral replication, transcription ${ }^{24}$ and packaging. ${ }^{25}$ In previous studies, antisense agents such as PNAs and phosphorodiamidate morpholino oligomers (PMOs) targeted to various sites in the 5' UTR of mouse hepatitis virus ${ }^{26}$ and SARS-CoV ${ }^{8 a, 27}$ were effectively antiviral. Recent research has shown that PMOs can effectively inhibit SARS-CoV-2 replication. ${ }^{28}$ To our knowledge, however, inhibition of SARS-CoV-2 with PNAs has not been reported. To further demonstrate the power of our automated single-shot technology, we synthesized an 8-member PPNA library targeting the 5'UTR, the transcription regulatory site (TRS), and the polyprotein $1 \mathrm{a} / \mathrm{b}$ translation start site (AUG) of SARS-CoV-2 within one day. After purification, each PPNA was obtained in milligram scale with $>90 \%$ purity (Fig. 5a).

Each synthetic PPNA was evaluated for its inhibitory effect on the replication of SARS-CoV-2 in Vero-E6 cell cultures. Anti-IVS2-654 PNA, which has no intracellular target present, was selected as a negative control, while the known inhibitor of viral growth, EK $1,{ }^{29}$ was used as a positive control. A multiplicity of infection (MOI) of 0.1 was used for virus inoculation for $2 \mathrm{~h}$. PPNA treatments were given at the same time as viral infection started and then continued after virus being removed. Subsequent viral RNA levels were measured $48 \mathrm{~h}$ after initial infection. We observed dose-dependent reduction of the viral RNA replication with increasing concentrations of PPNAs (Fig. 5b). Sequences designed to target the 5' UTR and AUG region were highly efficacious. The 5'UTR-3 sequence reduced titers by $75 \%$ in the live SARS-CoV-2 assay, which was at the same level as EK1. Nearly complete inhibition was achieved with sequence 5'UTR-1 at $10 \mu \mathrm{M}\left(\mathrm{IC}_{50}: 0.8 \mu \mathrm{M}\right.$. Fig. 5c). This result suggests that further study of the 5' UTR PPNAs to address SARS-CoV-2 infections is warranted, and the automated technology described here will provide a strong driving force for the development of this area. 
Concentration $(\mu \mathrm{M})$
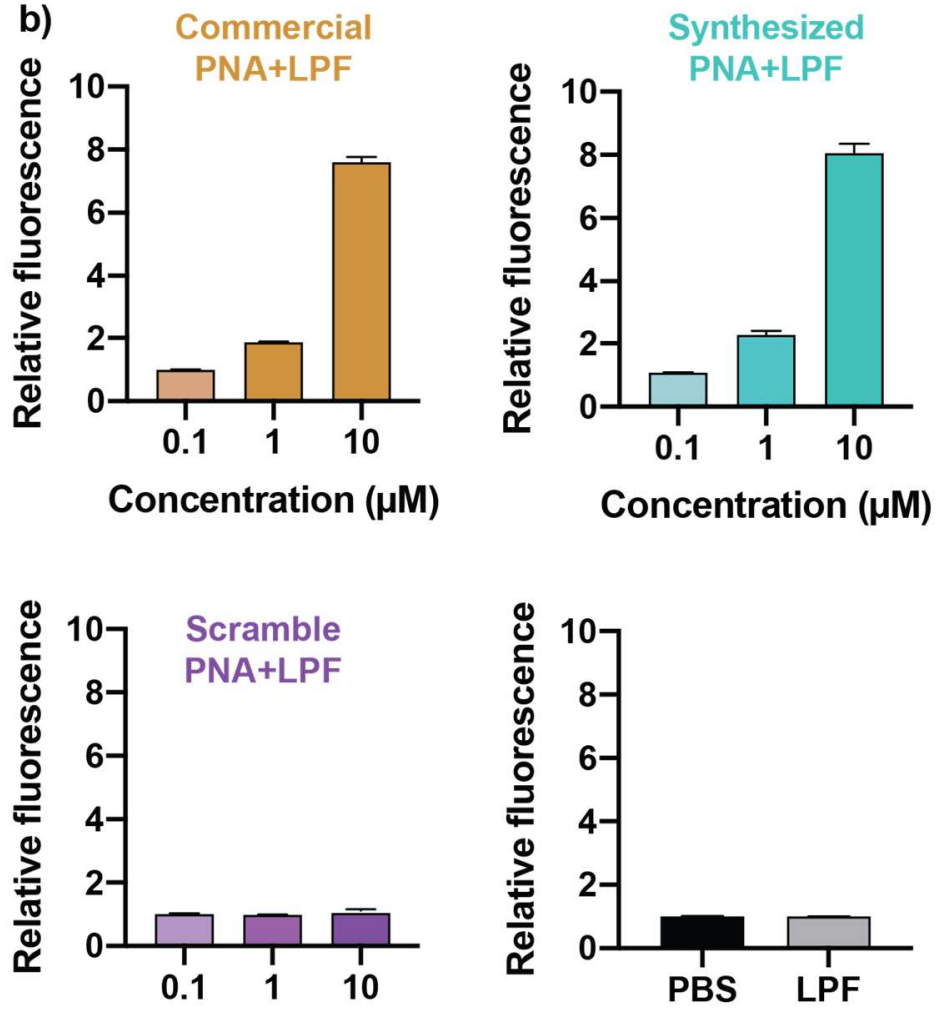

c)

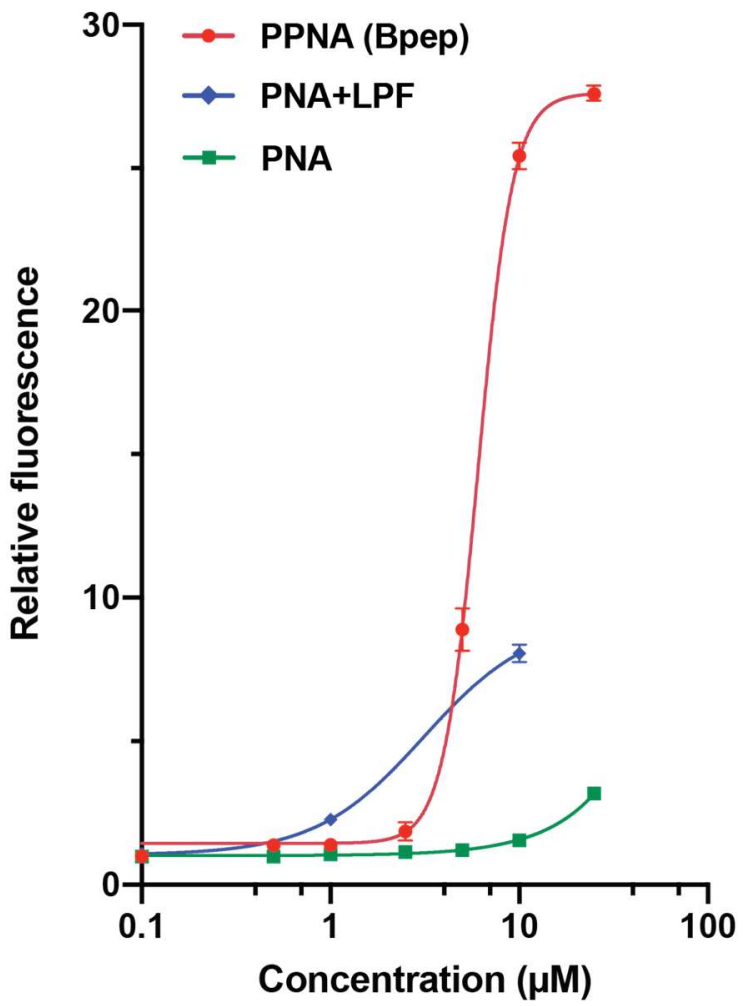

Figure 4. Automated flow-synthesized PNAs are highly active in live cell assays. (a) Names, sequences, quantities, and purities of EGFP PNAs and PPNA. (b) The relative fluorescence (to PBS vehicle-treated cells) of flow-synthesized anti-IVS2-654 PNA and relevant scramble PNA are compared to commercial anti-IVS2-654 PNA, as determined by an EGFP assay in HeLa-654 cells. PBS: Phosphate-Buffered Saline; LPF: Lipofectamine. (c) Dose-response curves corresponding to activity in the EGFP assay for synthesized unmodified PNA, PPNA, and PNA with lipofectamine. Activity is shown as fluorescence intensity relative to PBS vehicle-treated HeLa-654 cells.

\section{CONCLUSION}

The optimized automated flow PNA synthesis protocol demonstrates noticeable advantages of flow chemistry over traditional batch methods. A variable temperature design achieves a 10 -second amide bond formation while greatly suppressing side reactions. The merits of this automated technology are not only rapid synthesis with improved crude purity, but also the ability to access PNA sequences that are challenging to obtain using batch methods. More importantly, this methodology enables facile single-shot synthesis of PNA conjugates with cell-penetrating peptides (PPNAs), typically a time- and laborintensive task.

The automated strategy developed here overcomes the PNA production barrier and shifts the research focus in the field from synthesis to endpoint biological investigations and drug discovery. In our research, commercial PNAs (21-residue long) required a three-week lead time for preparation. With our technology, we prepared eight anti-SARS-CoV-2 PPNAs (33 residues each) in a single day. This is particularly important as a fast response to the current COVID-19 pandemic. We tested the viral inhibition of these flow-synthesized PPNAs in a live SARS-CoV-2 inhibition assay, and found that an important PPNA sequence targeting the 5'UTR region can reduce the viral titer by $>95 \%$. Given the favorable toxicological profile of PNA drugs, ${ }^{4 \mathrm{~b}}$ further improvements in efficacy could result in an effective treatment for SARS-CoV-2. Finally, this automated PNA synthesis protocol enables on-demand production of timesensitive and potentially life-saving personalized medicines, not only for COVID-19 but also for other genetic and acquired diseases and new viral pathogens. 


\begin{tabular}{c|ccccc}
\hline Entry & PPNA Name & Sequence $\left(5^{\prime} \rightarrow 3^{\prime}\right)$ & Location & Quantity & Purity \\
\hline 1 & anti-IVS2-654 & GCTATTACCTTAACCCAG-KKK-Bpep-CONH2 & - & $1.2 \mathrm{mg}$ & $>95 \%$ \\
2 & 5 'UTR-1 & TTGGTTGGTTTGTTACCT-KKK-Bpep-CONH2 & $21-39$ & $1.5 \mathrm{mg}$ & $>95 \%$ \\
3 & 5 'UTR-2 & AGTTACTCGTGTCCTGTC-KKK-Bpep-CONH2 & $155-172$ & $1.4 \mathrm{mg}$ & $>90 \%$ \\
4 & 5 'UTR-3 & CTTACCTTTCGGTCACAC-KKK-Bpep-CONH2 & $248-265$ & $1.3 \mathrm{mg}$ & $>90 \%$ \\
5 & TRS-1 & AAGTTCGTTTAGAGAACA-KKK-Bpep-CONH2 & $60-77$ & $0.9 \mathrm{mg}$ & $>95 \%$ \\
6 & TRS-2 & TTTTAAAGTTCGTTTAGA-KKK-Bpep-CONH2 & $65-82$ & $1.0 \mathrm{mg}$ & $>95 \%$ \\
7 & TRS-3 & AGATTTTAAAGTTCGTTT-KKK-Bpep-CONH2 & $68-85$ & $1.0 \mathrm{mg}$ & $>95 \%$ \\
8 & AUG & CTCTCCATCTTACCTTTC-KKK-Bpep-CONH2 & $254-271$ & $1.5 \mathrm{mg}$ & $>90 \%$
\end{tabular}

b)

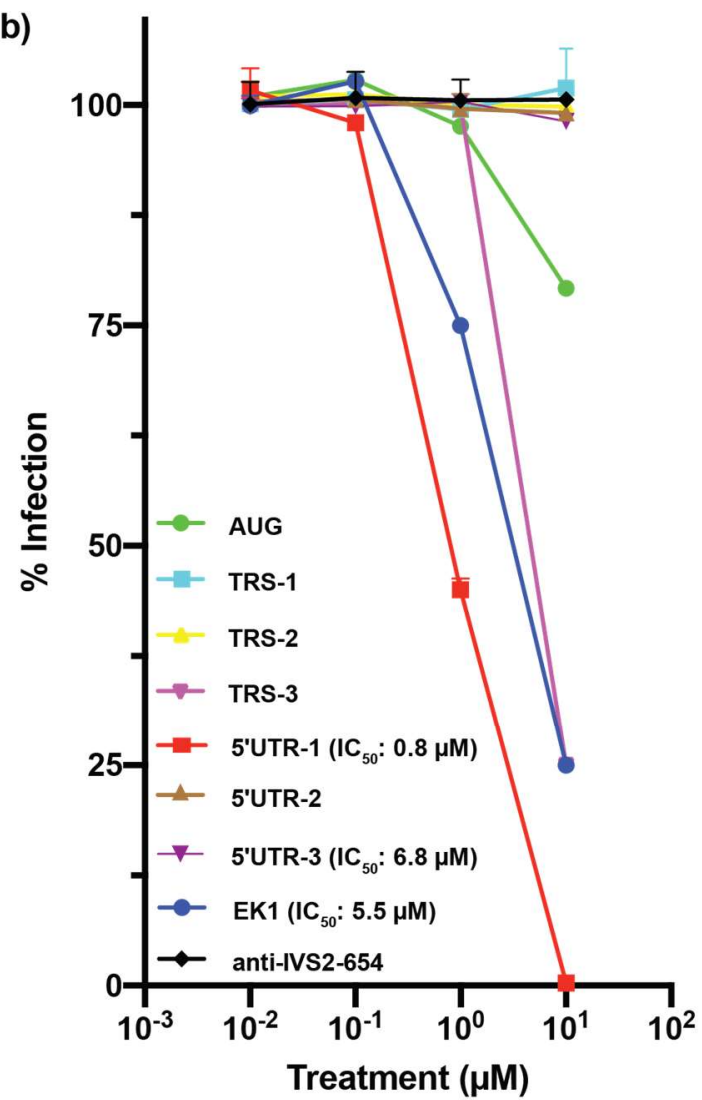

c)

\begin{tabular}{|l|l|l|}
\hline EK1 & 5'UTR-2 & TRS-2 \\
anti-IVS2-654 & 5'UTR-3 & TRS-3 \\
\hline 5'UTR-1 & TRS-1 & AUG \\
\hline
\end{tabular}

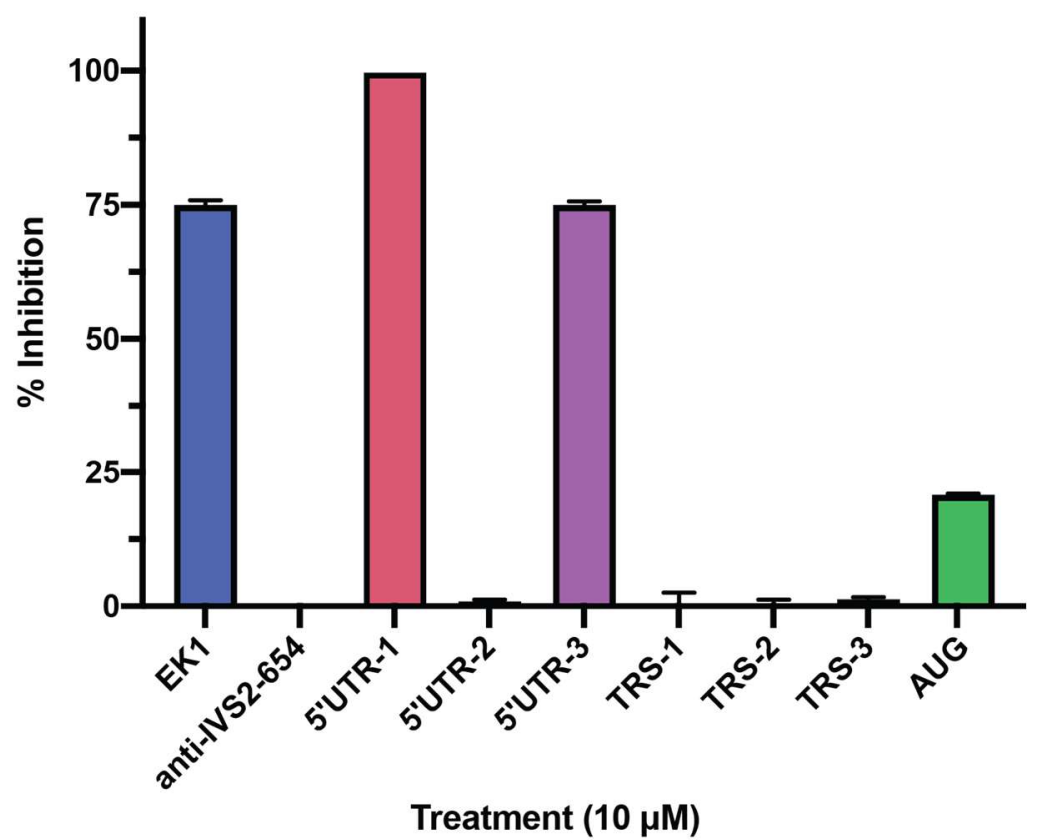

Figure 5. Dose-dependent inhibition of live SARS-CoV-2 replication by a synthetic PPNA library. (a) Names, sequences, target location, quantities, and purities of synthetic PPNAs. Locations on the SARS genomic RNA are based on GenBank NC 045512. Bases targeting the SARS-CoV-2 leader-TRS (nt 70-75) are in green; Bases targeting the AUG translation start site are in blue. (b) Increasing concentrations of PPNAs result in inhibition of viral replication as measured by total viral RNA present. EK1 as a positive control, and anti-IVS2-654 as a negative control. (c) Viral inhibition with PPNAs at $10 \mu \mathrm{M}$.

\section{ASSOCIATED CONTENT}

\section{Supporting information}

Experimental procedures and characterization data for all compounds, HPLC and LCMS traces, EGFP assay, and SARS$\mathrm{CoV}-2$ inhibition assays. The Supporting Information is available free of charge on the ACS Publications website at DOI: $10.1021 /$ jacs.

\section{AUTHOR INFORMATION}

\section{Corresponding Author}

Bradley L. Pentelute - Department of Chemistry, Massachusetts Institute of Technology, 77 Massachusetts Avenue, 
Cambridge, MA 02139, USA ; The Koch Institute for Integrative Cancer Research, Massachusetts Institute of Technology, 500 Main Street, Cambridge, MA 02142, USA ; Center for Environmental Health Sciences, Massachusetts Institute of Technology, 77 Massachusetts Avenue, Cambridge, MA 02139, USA ; Broad Institute of MIT and Harvard, 415 Main Street, Cambridge, MA 02142, USA; orcid.org/0000-0002-7242-801X; Email: blp@mit.edu

\section{Authors}

Chengxi Li - Department of Chemistry, Massachusetts Institute of Technology, 77 Massachusetts Avenue, Cambridge, MA 02139, USA ; orcid.org/0000-0003-3904-0299; Email: $\underline{\text { licx@mit.edu }}$

Alex J. Callahan - Department of Chemistry, Massachusetts Institute of Technology, 77 Massachusetts Avenue, Cambridge, MA 02139, USA ; orcid.org/0000-0002-8414-1382; Email: acallah@mit.edu

Kruttika-Suhas Phadke - Department of Veterinary Microbiology and Preventive Medicine, College of Veterinary Medicine, Iowa State University, Ames, IA 50011 USA ; orcid.org/00000002-0661-9520; Email: ksphadke@,iastate.edu

Bryan Bellaire - Department of Veterinary Microbiology and Preventive Medicine, College of Veterinary Medicine, Iowa State University, Ames, IA 50011 USA ; orcid.org/0000-00024034-6482; Email: bbella@iastate.edu

Charlotte E. Farquhar - Department of Chemistry, Massachusetts Institute of Technology, 77 Massachusetts Avenue, Cambridge, MA 02139, USA ; orcid.org/0000-0003-4919-2296; Email: farquhar@mit.edu

Genwei Zhang - Department of Chemistry, Massachusetts Institute of Technology, 77 Massachusetts Avenue, Cambridge, MA 02139, USA ; orcid.org/0000-0002-5737-8413; Email: genwei@mit.edu

Carly K. Schissel - Department of Chemistry, Massachusetts Institute of Technology, 77 Massachusetts Avenue, Cambridge, MA 02139, USA ; orcid.org/0000-0003-0773-5168; Email: schissel@mit.edu

Alexander J. Mijalis - Department of Chemistry, Massachusetts Institute of Technology, 77 Massachusetts Avenue, Cambridge, MA 02139, USA; Current address: Harvard Medical School, Department of Genetics, 77 Avenue Louis Pasteur, Boston, MA 02115, USA. Email: mijalis@hms.harvard.edu

Nina Hartrampf - Department of Chemistry, Massachusetts Institute of Technology, 77 Massachusetts Avenue, Cambridge, MA 02139, USA ; Current address: University of Zurich, Department of Chemistry, Wintherthurerstrasse 190, 8057 Zurich, Switzerland. orcid.org/ 0000-0003-0875-6390; Email: nina.hartrampf@chem.uzh.ch

Andrei Loas - Department of Chemistry, Massachusetts Institute of Technology, 77 Massachusetts Avenue, Cambridge, MA 02139, USA ; orcid.org/0000-0001-5640-1645; Email: loas@mit.edu

David E. Verhoeven - Department of Veterinary Microbiology and Preventive Medicine, College of Veterinary Medicine, Iowa State University, Ames, IA 50011 USA ; orcid.org/0000-00020766-4606; Email: davidver@iastate.edu

\section{Notes}

The Python code for automated operation of the flow synthesis instrument is available in a GitHub repository (http://doi.org/10.5281/zenodo.3774509).
B.L.P. is a co-founder of Amide Technologies and Resolute Bio. Both companies focus on developing protein and peptide therapeutics.

\section{ACKNOWLEDGMENT}

Financial support for this work was provided by Novo Nordisk A/S (to B.L.P.). A.J.C. is supported by the Koch Institute MIT School of Science Fellowship in Cancer Research. C.E.F. is supported by the National Science Foundation (NSF) Graduate Research Fellowship program (4000057441). We thank Andrew Wilson at Detailed Dynamic for help with the design and manufacture of the automated instrument.

\section{REFERENCES}

(1) (a) Nielsen, P. E.; Egholm, M.; Berg, R. H.; Buchardt, O. Sequence-Selective Recognition of DNA by Strand Displacement with a Thymine-Substituted Polyamide. Science 1991, 254, 1497-1500. (b) Siddiquee, S. A Review of Peptide Nucleic Acid. Adv. Tech. Bio. Med. 2015, 03, 1-10.

(2) Egholm, M.; Buchardt, O.; Christensen, L.; Behrens, C.; Freier, S. M.; Driver, D. A.; Berg, R. H.; Kim, S. K.; Norden, B.; Nielsen, P. E. PNA Hybridizes to Complementary Oligonucleotides Obeying the Watson-Crick Hydrogen-Bonding Rules. Nature 1993, 365, 566-568.

(3) Demidov, V. V.; Potaman, V. N.; Frank-Kamenetskil, M. D.; Egholm, M.; Buchard, O.; Sönnichsen, S. H.; Nlelsen, P. E. Stability of Peptide Nucleic Acids in Human Serum and Cellular Extracts. Biochem. Pharmacol. 1994, 48, 1310-1313.

(4) (a) Gupta, A.; Mishra, A.; Puri, N. Peptide Nucleic Acids: Advanced Tools for Biomedical Applications. J. Biotechnol. 2017, 259, 148-159. (b) Sharma, C.; Awasthi, S. K. Versatility of Peptide Nucleic Acids (PNAs): Role in Chemical Biology, Drug Discovery, and Origins of Life. Chem. Biol. Drug Des. 2017, 89, 16-37. (c) Montazersaheb, S.; Hejazi, M. S.; Nozad Charoudeh, H. Potential of Peptide Nucleic Acids in Future Therapeutic Applications. Adv. Pharm. Bull. 2018, 8, 551-563. (5) Alagpulinsa, D. A.; Yaccoby, S.; Ayyadevara, S.; Shmookler Reis, R. J. A peptide Nucleic Acid Targeting Nuclear RAD51 Sensitizes Multiple Myeloma Cells to Melphalan Treatment. Cancer Biol. Ther. 2015, 16, 976-986.

(6) Hoban, M. D.; Orkin, S. H.; Bauer, D. E. Genetic Treatment of a Molecular Disorder: Gene Therapy Approaches to Sickle Cell Disease. Blood 2016, 127, 839-848.

(7) Maekawa, K.; Azuma, M.; Okuno, Y.; Tsukamoto, T.; Nishiguchi, K.; Setsukinai, K.-i.; Maki, H.; Numata, Y.; Takemoto, H.; Rokushima, M. Antisense Peptide Nucleic Acid-Peptide Conjugates for Functional Analyses of Genes in Pseudomonas Aeruginosa. Biorg. Med. Chem. 2015, 23, 7234-7239.

(8) (a) Ahn, D.-G.; Lee, W.; Choi, J.-K.; Kim, S.-J.; Plant, E. P.; Almazán, F.; Taylor, D. R.; Enjuanes, L.; Oh, J.-W. Interference of Ribosomal Frameshifting by Antisense Peptide Nucleic Acids Suppresses SARS Coronavirus Replication. Antivir. Res. 2011, 91, 1-10. (b) Zeng, Z.; Han, S.; Hong, W.; Lang, Y.; Li, F.; Liu, Y.; Li, Z.; Wu, Y.; Li, W.; Zhang, X.; Cao, Z. A TatConjugated Peptide Nucleic Acid Tat-PNA-DR Inhibits Hepatitis B Virus Replication In Vitro and In Vivo by Targeting LTR Direct Repeats of HBV RNA. Mol. Ther. Nucleic Acids 2016, 5, e295.

(9) Wu, F.; Zhao, S.; Yu, B.; Chen, Y.-M.; Wang, W.; Song, Z.G.; Hu, Y.; Tao, Z.-W.; Tian, J.-H.; Pei, Y.-Y.; Yuan, M.-L.; Zhang, Y.-L.; Dai, F.-H.; Liu, Y.; Wang, Q.-M.; Zheng, J.-J.; $\mathrm{Xu}$, L.; Holmes, E. C.; Zhang, Y.-Z. A New Coronavirus 
Associated with Human Respiratory Disease in China. Nature 2020, 579, 265-269.

(10) Tailhades, J.; Takizawa, H.; Gait, M. J.; Wellings, D. A.; Wade, J. D.; Aoki, Y.; Shabanpoor, F. Solid-Phase Synthesis of Difficult Purine-Rich PNAs through Selective Hmb Incorporation: Application to the Total Synthesis of Cell Penetrating Peptide-PNAs. Front. Chem. 2017, 5 .

(11) (a) Angelone, D.; Hammer, A. J. S.; Rohrbach, S.; Krambeck, S.; Granda, J. M.; Wolf, J.; Zalesskiy, S.; Chisholm, G.; Cronin, L. Convergence of Multiple Synthetic Paradigms in a Universally Programmable Chemical Synthesis Machine. Nat. Chem. 2021, 13, 63-69. (b) Plante, O. J.; Palmacci, E. R.; Seeberger, P. H. Automated Solid-Phase Synthesis of Oligosaccharides. Science 2001, 291, 1523-1527. (c) Jiang, T.; Bordi, S.; McMillan, A. E.; Chen, K.-Y.; Saito, F.; Nichols, P.; Wanner, B.; Bode, J. W. An Integrated Console for Capsule-Based, Fully Automated Organic Synthesis. ChemRxiv 2019, doi.org/10.26434/chemrxiv.7882799.v1.

(12) Mayfield, L. D.; Corey, D. R. Automated Synthesis of Peptide Nucleic Acids and Peptide Nucleic Acid-Peptide Conjugates. Anal. Biochem. 1999, 268, 401-404.

(13) Braasch, D. A.; Nulf, C. J.; Corey, D. R. Synthesis and Purification of Peptide Nucleic Acids. Curr. Protoc. Nucleic Acid Chem. 2002, 9, 4.11.11-14.11.18.

(14) Gogoi, K.; Mane, M. V.; Kunte, S. S.; Kumar, V. A. A Versatile Method for the Preparation of Conjugates of Peptides with DNA/PNA/Analog by Employing Chemo-Selective Click Reaction in Water. Nucleic Acids Res. 2007, 35, e139.

(15) Rostovtsev, V. V.; Green, L. G.; Fokin, V. V.; Sharpless, K. B. A Stepwise Huisgen Cycloaddition Process: Copper(I)Catalyzed Regioselective "Ligation" of Azides and Terminal Alkynes. Angew. Chem. Int. Ed. 2002, 41, 2596-2599.

(16) Sazani, P.; Kang, S.-H.; Maier, M. A.; Wei, C.; Dillman, J.; Summerton, J.; Manoharan, M.; Kole, R. Nuclear Antisense Effects of Neutral, Anionic and Cationic Oligonucleotide Analogs. Nucleic Acids Res. 2001, 29, 3965-3974.

(17) Li, C.; Callahan, A. J.; Simon, M. D.; Totaro, K. A.; Mijalis, A. J.; Hartrampf, N.; Schissel, C. K.; Zhou, M.; Zong, H.; Hanson, G. J.; Loas, A.; Pohl, N. L. B.; Pentelute, B. L. Automated Fast-Flow Synthesis of Antisense Phosphorodiamidate Morpholino Oligomers. ChemRxiv 2020. doi.org/10.26434/chemrxiv.12685886.v1.

(18) Mijalis, A. J.; Thomas III, D. A.; Simon, M. D.; Adamo, A.; Beaumont, R.; Jensen, K. F.; Pentelute, B. L. A Fully Automated Flow-Based Approach for Accelerated Peptide Synthesis. Nat. Chem. Biol. 2017, 13, 464-466.

(19) Hartrampf, N.; Saebi, A.; Poskus, M.; Gates, Z. P.; Callahan, A. J.; Cowfer, A. E.; Hanna, S.; Antilla, S.; Schissel, C. K.; Quartararo, A. J.; Ye, X.; Mijalis, A. J.; Simon, M. D.; Loas, A.; Liu, S.; Jessen, C.; Nielsen, T. E.; Pentelute, B. L. Synthesis of Proteins by Automated Flow Chemistry. Science 2020, 368, 980-987.

(20) Egholm, M.; Buchardt, O.; Nielsen, P. E.; Berg, R. Peptide Nucleic Acids (PNA). Oligonucleotide Analogues with an Achiral Peptide Backbone. J. Am. Chem. Soc. 1992. 144, 18951897.
(21) (a) Pooga, M.; Soomets, U.; Hällbrink, M.; Valkna, A.; Saar, K.; Rezaei, K.; Kahl, U.; Hao, J.-X.; Xu, X.-J.; Wiesenfeld-Hallin, Z.; Hökfelt, T.; Bartfai, T.; Langel, Ü. Cell Penetrating PNA Constructs Regulate Galanin Receptor Levels and Modify Pain Transmission in Vivo. Nat. Biotechnol. 1998, 16, 857-861. (b) Gait, M. J.; Arzumanov, A. A.; McClorey, G.; Godfrey, C.; Betts, C.; Hammond, S.; Wood, M. J. A. Cell-Penetrating Peptide Conjugates of Steric Blocking Oligonucleotides as Therapeutics for Neuromuscular Diseases from a Historical Perspective to Current Prospects of Treatment. Nucleic Acid Ther. 2018, 29, 1-12.

(22) Fadzen, C. M.; Holden, R. L.; Wolfe, J. M.; Choo, Z. N.; Schissel, C. K.; Yao, M.; Hanson, G. J.; Pentelute, B. L. Chimeras of Cell-Penetrating Peptides Demonstrate Synergistic Improvement in Antisense Efficacy. Biochemistry 2019, 58, 3980-3989.

(23) Simon, M. D.; Heider, P. L.; Adamo, A.; Vinogradov, A. A.; Mong, S. K.; Li, X.; Berger, T.; Policarpo, R. L.; Zhang, C.; Zou, Y.; Liao, X.; Spokoyny, A. M.; Jensen, K. F.; Pentelute, B. L. Rapid Flow-Based Peptide Synthesis. ChemBioChem 2014, 15, 713-720.

(24) Madhugiri, R.; Fricke, M.; Marz, M.; Ziebuhr, J. RNA Structure Analysis of Alphacoronavirus Terminal Genome Regions. Virus Res. 2014, 194, 76-89.

(25) Masters, P. S. Coronavirus Genomic RNA Packaging. Virology 2019, 537, 198-207.

(26) (a) Burrer, R.; Neuman, B. W.; Ting, J. P. C.; Stein, D. A.; Moulton, H. M.; Iversen, P. L.; Kuhn, P.; Buchmeier, M. J. Antiviral Effects of Antisense Morpholino Oligomers in Murine Coronavirus Infection Models. J. Virol. 2007, 81, 5637-5648. (b) Neuman, B. W.; Stein, D. A.; Kroeker, A. D.; Paulino, A. D.; Moulton, H. M.; Iversen, P. L.; Buchmeier, M. J. Antisense Morpholino-Oligomers Directed against the $5^{\prime}$ End of the Genome Inhibit Coronavirus Proliferation and Growth. J. Virol. 2004, 78, 5891-5899.

(27) Neuman, B. W.; Stein, D. A.; Kroeker, A. D.; Churchill, M. J.; Kim, A. M.; Kuhn, P.; Dawson, P.; Moulton, H. M.; Bestwick, R. K.; Iversen, P. L.; Buchmeier, M. J. Inhibition, Escape, and Attenuated Growth of Severe Acute Respiratory Syndrome Coronavirus Treated with Antisense Morpholino Oligomers. $J$. Virol. 2005, 79, 9665-9676.

(28) Rosenke, K.; Leventhal, S.; Moulton, H. M.; Hatlevig, S.; Hawman, D.; Feldmann, H.; Stein, D. A. Inhibition of SARSCoV-2 in Vero cell cultures by peptide-conjugated morpholino oligomers. J. Antimicrob. Chemother. 2021, 76, 413-417.

(29) (a) Xia, S.; Yan, L.; Xu, W.; Agrawal, A. S.; Algaissi, A.; Tseng, C.-T. K.; Wang, Q.; Du, L.; Tan, W.; Wilson, I. A.; Jiang, S.; Yang, B.; Lu, L. A Pan-Coronavirus Fusion Inhibitor Targeting the HR1 Domain of Human Coronavirus Spike. Sci. Adv. 2019, 5, eaav4580. (b) Xia, S.; Liu, M.; Wang, C.; Xu, W.; Lan, Q.; Feng, S.; Qi, F.; Bao, L.; Du, L.; Liu, S.; Qin, C.; Sun, F.; Shi, Z.; Zhu, Y.; Jiang, S.; Lu, L. Inhibition of SARS-CoV-2 (Previously 2019-nCoV) Infection by a Highly Potent PanCoronavirus Fusion Inhibitor Targeting Its Spike Protein that Harbors a High Capacity to Mediate Membrane Fusion. Cell Res. 2020, 30, 343-355. 
Table of Contents Graphic
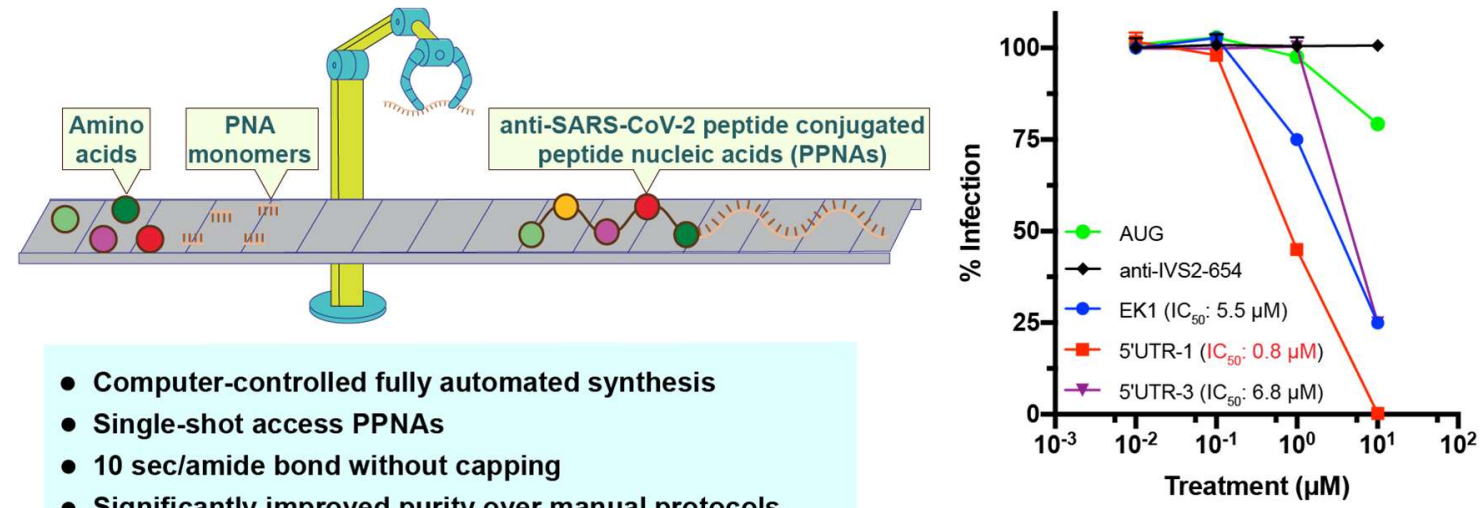

- Computer-controlled fully automated synthesis

- Single-shot access PPNAs

- 10 sec/amide bond without capping

- Significantly improved purity over manual protocols

Over 95\% SARS-CoV-2 inhibition 